\title{
Management of Orthopaedic Patients During COVID-19 Pandemic in India: A Guide
}

\author{
Deepak Neradi ${ }^{1} \cdot$ Aman Hooda $^{1} \cdot$ Akshay Shetty $^{1} \cdot$ Deepak Kumar $^{1} \cdot$ Amit Kumar Salaria $^{1} \cdot$ Vijay Goni $^{1}$
}

Received: 4 April 2020 / Accepted: 15 April 2020 / Published online: 27 April 2020

(c) Indian Orthopaedics Association 2020

\section{Introduction}

Coronaviruses are a group of viruses that mainly affect human beings through animal transmission. It is the third time, the emergence of novel coronavirus in last two decades, Severe acute respiratory syndrome (SARS) in 2003 [1], Middle East respiratory syndrome coronavirus (MERS$\mathrm{CoV}$ ) in 2012 [2] and novel severe acute respiratory syndrome coronavirus (SARS-CoV-2)-infected pneumonia (COVID-19). The novel coronavirus first emerged in Wuhan, China in December 2019 from the wet seafood market [3]. COVID-19 was regarded as a public health emergency of international concern in the world by mid-February 2019 [4]. The epicentre of the pandemic shifted from Europe to USA from time to time and at present there are around 17.8 lakhs cases of COVID-19 with 109,275 casualties in the world, amounting to $6.12 \%$ mortality rate according to World Health Organization (WHO) as of now. The number of cases and deaths are increasing day by day and the infection is spreading to almost every corner of this world.

India is a developing country with around 1.3 billion population, 2nd largest in the world after China. In India, there is one allopathic doctor per 10,926 population [5], which is below WHO's recommendation of 1:1000 [6], putting

\author{
Aman Hooda \\ amanhooda_10@yahoo.com \\ Deepak Neradi \\ n.deepak47@gmail.com \\ Akshay Shetty \\ akshayshettyt@live.com \\ Deepak Kumar \\ drdeepaknegimt@gmail.com \\ Amit Kumar Salaria \\ meetamit20salaria@gmail.com \\ Vijay Goni \\ vijaygoni@gmail.com \\ 1 Department of Orthopaedics, PGIMER, Chandigarh, India
}

tremendous pressure on the health care system in India due to COVID-19. The first case of COVID-19 was reported on 30th January 2020 and the number has reached 8500 as on 12th April 20, with 289 deaths. On 25th March 2020, Prime minister of India announced a nationwide 3-week lockdown to prevent community transmission in India. This lockdown has been extended further and we have no idea when this lockdown gets released. Even after the release of lockdown, the situation will not be the same as in the past and we have to be more careful in attending patients.

The hospitals are becoming hot zones for the treatment as well as transmission of COVID-19 due to a rise in the community transmission from Europe, Asia and the rest of the world. Orthopaedic surgeries including both elective and emergency procedures (trauma patients) require operation theatres which are high-risk areas for transmission of COVID-19, risks health care workers contracting this illness and decreasing the resources available to the population of India during this pandemic. The high prevalence of COVID19 , limited resources and staff, increased risks of transmission and the burden on health systems during this pandemic; keeping all this in mind, the health system must act immediately and support essential surgical care while protecting patients and staff and conserving valuable resources.

\section{Orthopaedic Patients Expected During Lockdown Period}

1. Trauma.

2. History of fall at home, the neck of femur fracture in elderly.

3. History of assault.

4. Severe cervical or lumbar pain.

5. Post-operative cases for wound dressing or suture removal.

6. Postoperative surgical site infections.

7. Elective cases with severe symptoms. 


\section{Follow These Steps to Create a Safe Working Environment}

\section{Ensure Safe Working Environment}

The examination area in the emergency especially door handles, working stations and frequently used items should be cleaned regularly at least four times a day with $1 \%$ hypochlorite/lysol. Ensure that the healthcare staff including the doctor, nurses and paramedical staff have no signs and symptoms related to COVID-19 infection or any contact with COVID patients in the past 14 days and it is better to screen the health care staff, if feasible. All health care staff should wear a personal protective equipment (PPE) in the emergency, if not at least wear an N-95 mask, a surgical gown and examination gloves and shoe covers. Education of health care staff, patients and their attendants should be of utmost priority.

\section{How to Attend Patients?}

A three-layer surgical mask, hand sanitizer and a disposable glove box should be available at the entry point of the emergency area for patients and their attendants. In case of trauma, it might be not possible to wear a mask for the patient in all cases, at least ensure that attendants are provided with one. History of COVID-19 like symptoms and any history of contact should be obtained both from patient and attendant and a separate perform should be attached to record all information. If there is any positive history then isolate both patient and attendant and treat as COVID positive unless proved otherwise. It is better to keep every patient in isolation and convert every ward to isolation rooms as there will be a limited number of patients in inpatient departments (IPD). Maintain a separate dressing room and plaster room for patients and waste like dressing material, gauges etc. of suspected patients should be disposed of carefully.

\section{Avoid Negligence Towards Elective Patients with Severe Symptoms}

Every symptom should be recorded carefully and one should not be negligent towards elective patients. Patients with tumour or pathological fracture, or cauda equina or any infection should be investigated properly and surgical intervention should be deferred unless it is required on an urgent basis. We may also have cases like avascular necrosis/ ankylosing hips or rheumatoid knee where patients present with severe pain, adequate analgesia should be given to get rid of acute symptoms.

\section{How to Manage a Trauma Patient with COVID-19-Like Symptoms? (Having Signs or History of Contact)}

Inform hospital administration authority, CMO or SMO. A specialized COVID area in the triage should be ready for COVID-19 patients with trauma. Resuscitate the patient with a primary survey along with splintage of fracture limb. All necessary pre-operative investigations along with COVID19 testing should be done. If possible, get portable X-rays and ultrasound to avoid contamination of the radiology area and it also helps in decreasing movement of COVID patients. For investigations like CT scan or MRI, we have to sterilize the respective area after investigating every patient as per centres for disease control and prevention guidelines $[7,8]$. Patients with closed fractures should wait for surgical interventions until the COVID-19 results are out. All cases which need urgent management like an open fracture, vascular injuries, compartment syndrome or mangled limb; we cannot wait until COVID results. These patients should be managed as COVID positive patients and strict precautions should be taken to avoid transmission to caregivers or to other patients. If the results are positive keep the patient in the COVID isolation ward until the results are negative and take the help of the COVID response team of the hospital. If the results are negative shift the patient to the orthopaedic ward and then discharge as early as possible.

We depicted these management protocols in our flowchart below. We have postulated guidelines for management of non COVID patients (standard protocol) and COVID positive patients (COVID protocol). 
Patient in triage with an

Orthopaedic emergency

igh index of suspicion.Treat

every patient as COVID positive unless and until proven symptoms of COVID-19 and history of any contact

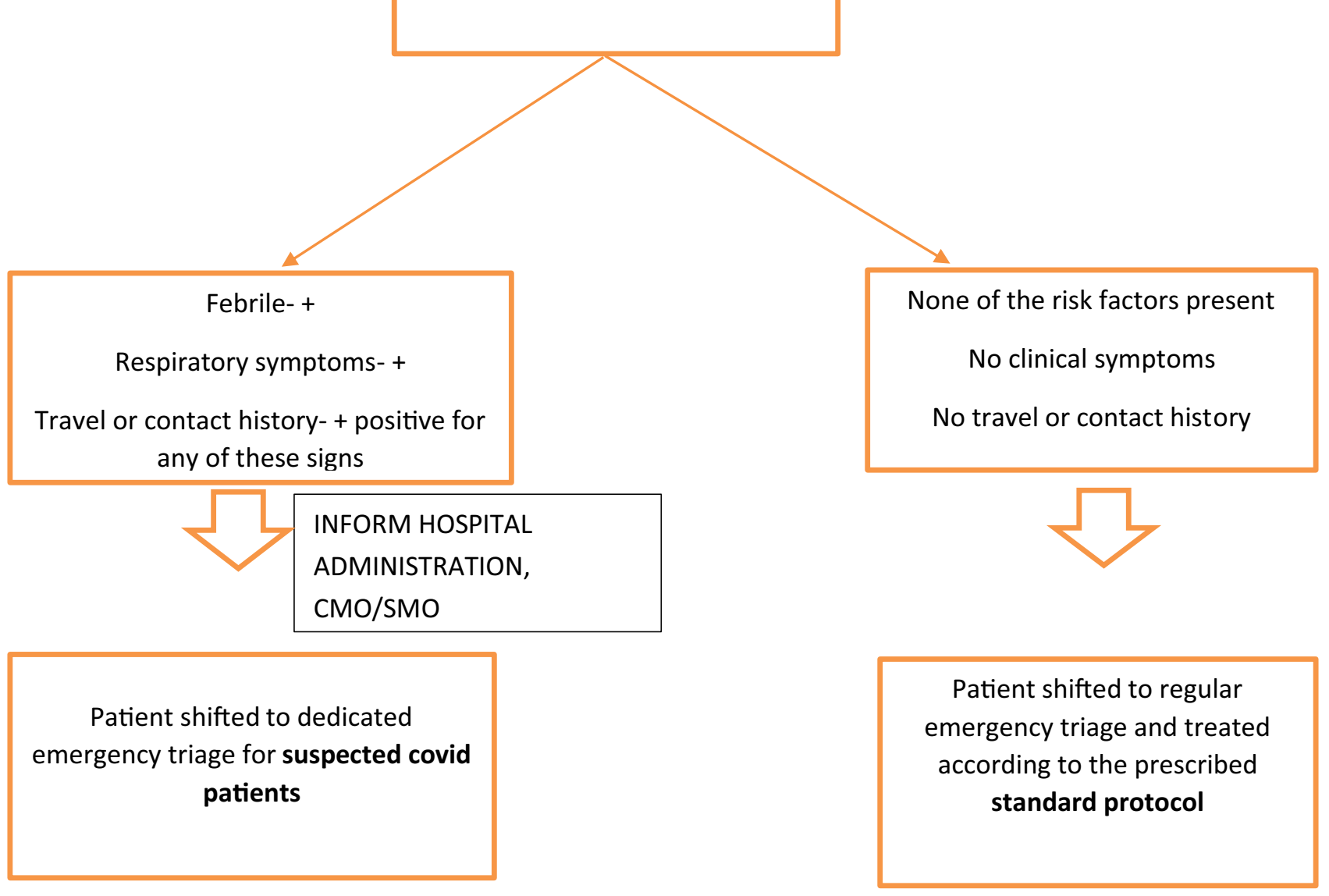


Test the patient for all preoperative investigations and COVID-19

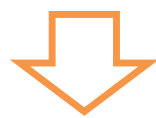

Fractures which can be waited for management (closed fractures)

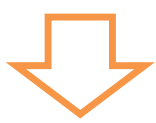

Wait for test results

POSITIVE

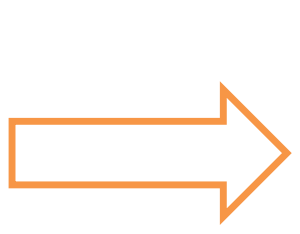

Fractures which are supposed to be managed on emergency basis like, open fractures, fracture with vascular injuries, compartment syndrome, mangled limbs etc.

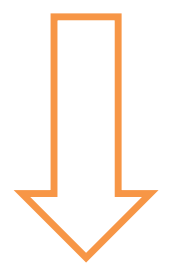

Treat as COVID protocol

\section{NEGATIVE}

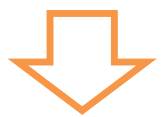

Treat as standard protocol 


\section{Standard Protocol}

1. Resuscitate patient, rule out all other injuries (Primary survey).

2. High chances of missed injuries in light of COVID suspicion (Secondary survey).

3. Manage conservatively whenever possible.

4. Keep patients in isolation wards. Provide patients and attendants with masks. Minimize patient and attendants' movements. Expedite the process of operation and discharge to lessen the load over the health system. These patients should be attended by separate team surgeons.

5. Maintain a follow up OPD in a separate area for dressing, suture removal and Plaster removal.

\section{Covid Protocol}

1. Manage conservatively whenever possible.

2. From the triage area patients (separate allocated area for COVID) should be shifted to the operating room.

3. Strict regulations must be maintained while shifting the patients. Sterilize all things that used while shifting, viz. trolley, lift etc.

4. Maintain a dedicated COVID operating room with trained staff.

5. Preventive measures must be followed at every level.

6. Every effort should be made to minimize the duration of surgery.

7. Decrease blood spilling.

8. Proper disposal of surgical waste.

9. Maintain negative pressure ventilation.

10. Patients have to be shifted to dedicated COVID isolation wards postoperatively and discharged only after COVID results are negative.

11. Care must be taken during the hospital stay to physiotherapy, bedsores and DVT prevention.

\section{Discussion}

Patients presented to the emergency triage with an orthopaedic emergency such as joint dislocations, compartment syndrome, open fractures, mangled extremity, polytrauma with FESS should be managed according to a specific guideline during global health emergencies like a pandemic of COVID-19. These orthopaedic emergencies require effective outpatient, inpatient and surgical care besides avoiding transmission of infection to fellow patients and health care givers. Low- and middle-income countries in Southeast Asia require a standard protocol that can be followed throughout the country with minimum resources available to ease burden over the health care system. There are no guidelines published in the past. Hence, this article can be valuable for the development of a standard universal guidelines for management these emergencies.

The patient attendees should also be screened for the risk factors and number of visitors to be restricted. Contact tracing can also be done with the help of these visitors. The department of Preventive and Social Medicine and COVID response team should be involved in this regard.

To prevent cross-contamination among fellow residents and faculty, it's imperative to have a dedicated orthopaedic team to manage these suspected or diagnosed COVID-19 patients. This team should comprise of a junior resident, registrar and consultant. This team has to manage and follow these patients throughout their hospital stay including the pre-operative, intra-operative and post-operative care. They are not allowed to attend other patients and remain segregated from the other department colleagues. We need to have $2-3$ such teams who work according to shifts. They should be advised to wear a triple layer surgical mask (preferably N-95) and hand hygiene to be maintained with the use of hand sanitizers and frequent hand washing. They must wear full PPE and should be taught how to wear and remove PPE effectively.

The lesson learned worldwide by orthopaedic surgeons can benefit India, to stay on top as we plan our approach to orthopaedic surgery during this pandemic of COVID-19. One should always remember that we are a doctor before an Orthopaedician. We should collectively work with other departments to face this pandemic.

Acknowledgements The guidelines provided here are not truly evidence based. These are based on individual opinion and experience of our seniors, besides some information available from guidelines by other expert bodies.

\section{Compliance with Ethical Standards}

Conflict of interest The authors declare that they have no conflict of interest.

Ethical standard statement This article does not contain any studies with human or animal subjects performed by any of the authors.

Informed consent For this type of study informed consent is not required.

\section{References}

1. Ramadan, N., \& Shaib, H. (2019). Middle East respiratory syndrome coronavirus (MERS-CoV): A review. Germs, 9, 35-42.

2. Zhong, N. S., Zheng, B. J., Li, Y. M., Poon, X. Z. H., Chan, K. H., et al. (2003). Epidemiology and cause of severe acute respiratory syndrome (SARS) in Guangdong, People's Republic of China. Lancet, 362, 1353-1358. 
3. Enserink M. Update: 'A bit chaotic.' Christening of new coronavirus and its disease name create confusion. https://www.scien cemag.org/news/2020/02/bit-chaoticchristening-new-coronaviru s-and-its-disease-name-createconfusion. Accessed 16 Feb 2020.

4. World Health Organization. Statement on the Second Meeting of the International Health Regulations. Emergency Committee regarding the outbreak of novel coronavirus (2019-nCoV). 2005. https://www.who.int/news-room/detail/30-01-2020-statementon-the-secondmeeting-of-the-international-health-regulation s-(2005)-emergency-committee-regarding-the-outbreak-of-novel coronavirus-(2019-ncov). Accessed 17 Feb 2020.

5. DelhiNovember $1 \mathrm{PT}$ of IN, November 1 2019UPDATED: Ist 2019 00:00. Only one allopathic govt doctor for 10,926 people in India: Report [Internet]. India Today. https://www.indiatoday.in/ india/story/allopathic-government-doctor-1614589-2019-11-01. Accessed 12 Apr 2020
6. Kumar, R., \& Pal, R. (2018). India achieves WHO recommended doctor population ratio: A call for paradigm shift in public health discourse! J Fam Med Prim Care., 7(5), 841-844.

7. Mossa-Basha, M., Meltzer, C. C., Kim, D. C., Tuite, M. J., Kolli, K. P., \& Tan, B. S. (2020). Radiology department preparedness for COVID-19: Radiology scientific expert panel. Radiology, 16, 200988.

8. https://www.cdc.gov/hai/pdfs/resource-limited/environmentalcleaning

Publisher's Note Springer Nature remains neutral with regard to jurisdictional claims in published maps and institutional affiliations. 